\title{
A Cytohistopathological Correlation of Thyroid Lesions with Critical Evaluation Of Discordant Cases: An Experience At A Tertiary Care Hospital
}

\author{
Shakuntala Sunil Aramani ${ }^{1 *}$ and Gururajaprasad. C ${ }^{2}$ \\ ${ }^{1}$ Department of Pathology, Gadag Institute of Medical Sciences, Mallasamudra, Gadag-Karnataka, India. \\ ${ }^{2}$ Department of Pathology, JSS Medical College, Mysuru, Karnataka, India.
}

\begin{abstract}
Background: Fine Needle Aspiration Cytology (FNAC) is important for pre-operative diagnosis of benign and malignant thyroid lesions. It is usually the first line of investigation and a minimally invasive diagnostic procedure whose essential role is to diagnose and distinguish benign from malignant lesions. Thus the present study was undertaken to determine sensitivity, specificity and accuracy of FNAC in the diagnosis of thyroid lesions and to correlate cytological findings with histopathology.

Methods: A prospective study was conducted in a tertiary care centre to study FNAC of thyroid lesions along with its histopathological correlation over a period of 2 years starting from June 2013 to June 2015

Result: $56(93 \%)$ patients were females and 4(7\%) patients were males. Patients age ranged from 15 to 65 years .Commonest lesion encountered in thyroid gland was colloid goitre, while papillary carcinoma was the most frequent malignant lesion. Cyto-histopathological correlation of 60 cases was done. Sensitivity ,specificity and diagnostic accuracy of the study for malignant lesions was $96.36 \%, 100 \%$,and $96.66 \%$ respectively.

Conclusion: FNAC is a safe, simple, highly accurate, economical method for evaluation of palpable thyroid lesions. There is almost perfect cyto-histopathological concordance and the results are consistent with those available in the literature. FNAC helps in avoiding unnecessary surgeries in patients diagnosed to have a benign pathology based on cytology. Thus FNAC serves as a useful screening test to triage thyroid lesions before surgery.
\end{abstract}

\section{Keywords: Thyroid, Histopathological Correlation, FNAC, Sensitivity, Specificity, Diagnostic accuracy}

\section{Introduction}

Head and neck lesions are very common and they range from inflammatory lesions to malignancies. It is evident that early diagnosis of these lesions provide the best chance of successful treatment. Among head and neck lesions thyroid lesions are most common with a prevalence rate ranging from $4-7 \% \cdot{ }^{[1]}$ The majority of clinically diagnosed thyroid lesions are non-neoplastic ;only 5-30\%,are malignant and require surgical intervention. ${ }^{[2]}$

FNAC is a minimally invasive procedure which is safe, simple, cost effective reliable and produces a quick result. ${ }^{[3]}$ FNAC has allowed a dramatic decrease in unnecessary surgeries without thyroid nodular disease, enhancing the percentage of malignant operated nodules over $50 \%$. ${ }^{[4]}$ Therefore the main aim of this diagnostic procedure in thyroid lesions is to detect the thyroid neoplasms for surgical resection and to identify non neoplastic lesions that may be managed conservatively.
Thus, this study was undertaken to study the cytology of various palpable thyroid lesions and also confirmation of the diagnosis by histopathological study.

\section{Materials and Methods}

The present study was a prospective, cross-sectional study which was undertaken to study FNAC of thyroid lesions along with its histopathological correlation at department of pathology , JSS Medical college, Mysuru over a period of 2 years from June 2013 to June 2015 .

All the patients were clinically examined in detail, and a careful palpation of the thyroid gland was done to judge precisely the location for aspiration. After brief explanation about the procedure to the patient multiple passes (2 -4) were done under aseptic precaution using a 24-25 gauge needle using a $10 \mathrm{ml}$ syringe. Multiple smears were prepared from aspirate and those immediately fixed in 95\%ethanol were stained using Haematoxylin and Eosin (H \& E) and Papanicolaou(Pap) stains and air dried smears were stained with May Grunwald Giemsa (MGG). 
Whenever the aspirate yielded fluid, it was cytocentrifused and the smears were prepared from the sediment and stained by the stains described earlier and the residual solid mass was sampled. The cases were followed by postoperative specimen received from the department of Surgery, department of ENT. Specimens were collected in $10 \%$ formalin in fresh state and allowed to fix for 24 hours. Detailed gross examination was done and bits were given. Paraffin embedded H\&E stained sections were obtained and studied under light microscopy. All the patients who presented with palpable thyroid lesions and who underwent FNAC with subsequent histopathological examination were included in the study and those patients in whom FNAC was either acellular or non diagnostic and who underwent FNAC without subsequent histopathological examination were excluded from the study. Statistical Package for Social Sciences(SPSS) software was used for statistical analysis. Sensitivity, specificity, and diagnostic accuracy(efficacy) were calculated.

Cytological diagnosis was correlated with the histopathology and the efficacy of FNAC is estimated by using the methodology of Galen and Gambino. These statistical values are interdependent statistical concepts indicating the accuracy of results.

\section{Results}

The present study includes a total number of 60 cases who presented with thyroid lesions. Patients age ranged from 15 to 65years with predominance of female patients with F:M ratio being 14:1. Graph-1 shows distribution of thyroid lesions with regard to sex, whereas Table-1 shows age and sex wise distribution of thyroid lesions. Majority of the patients are in the age group of 21-40 years followed by 41-60years.

In the present study, the non neoplastic lesions were more common then neoplastic lesions

Colloid goitre: Out of 60 cases, 47 cases were diagnosed as colloid goitre by cytology. Histopathological study confirmed the cytodiagnosis in 40 cases. In the remaining 7 cases, 5 cases turned out be follicular adenoma, 1 case as follicular carcinoma, and the other 1 case as papillary carcinoma.

Follicular neoplasm: Out of 60 cases, 7 cases were diagnosed as follicular neoplasm by cytology. 5 cases were confirmed by histopathology and the rest 2 cases turned out to be colloid goitre. Gross picture of follicular adenoma is shown in the fig-1,whereas fig-2 and the fig-3 shows the cytology of follicular neoplasm and histopathological section of follicular adenoma respectively.

Hashimoto's thyroiditis: 1 case was diagnosed as hashimoto's thyroiditis which was confirmed by histopathology.

Papillary carcinoma: Out of 60 cases, 5 cases were diagnosed as papillary carcinoma and all the cases were confirmed by histopathology.

Of the 60 cases, 55 cases were diagnosed as benign and only 5 cases turned out to be malignant by FNAC, where as 53 cases were diagnosed as benign and 7 cases turned out to be malignant by histopathology resulting in 2 false negative cases. The present study showed $96.36 \%$ sensitivity, $100 \%$ specificity and $96.66 \%$ diagnostic accuracy in detecting malignant tumors.

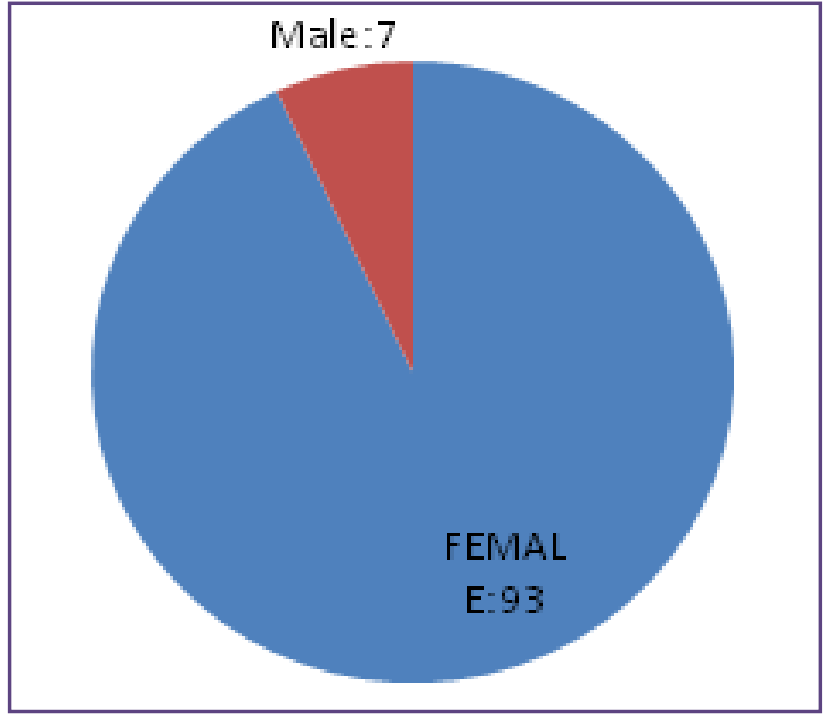

Graph 1: Distribution of thyroid lesion with regard to sex $(n=60)$ 


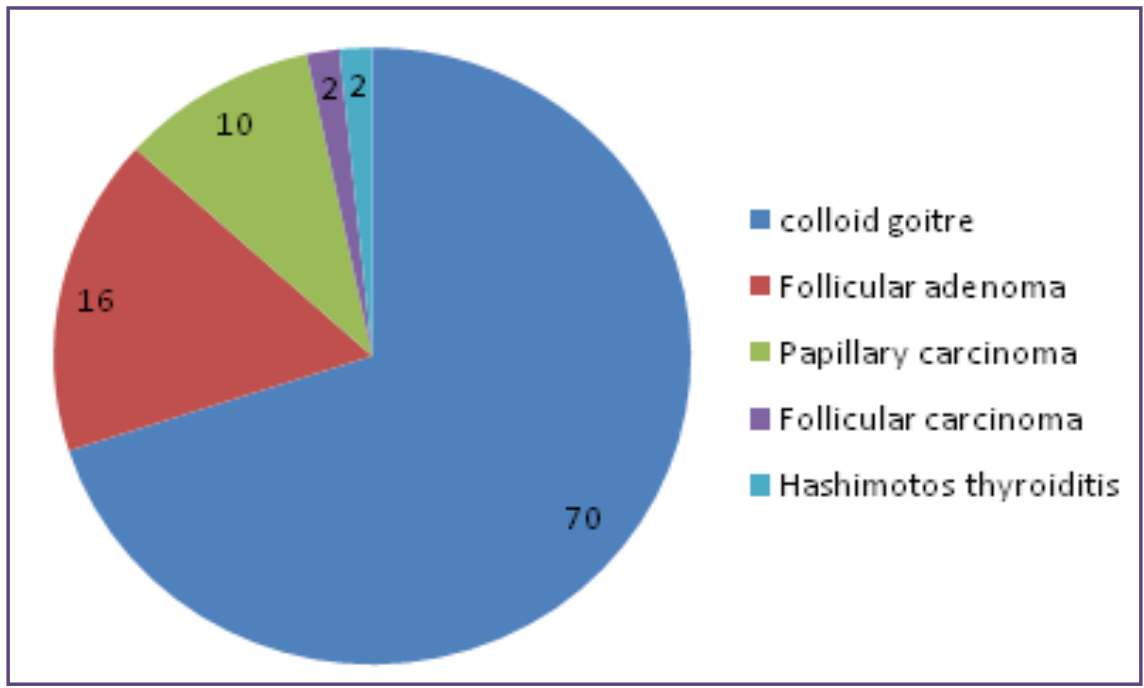

Graph 2: The various histopathological diagnosis of the thyroid lesions.

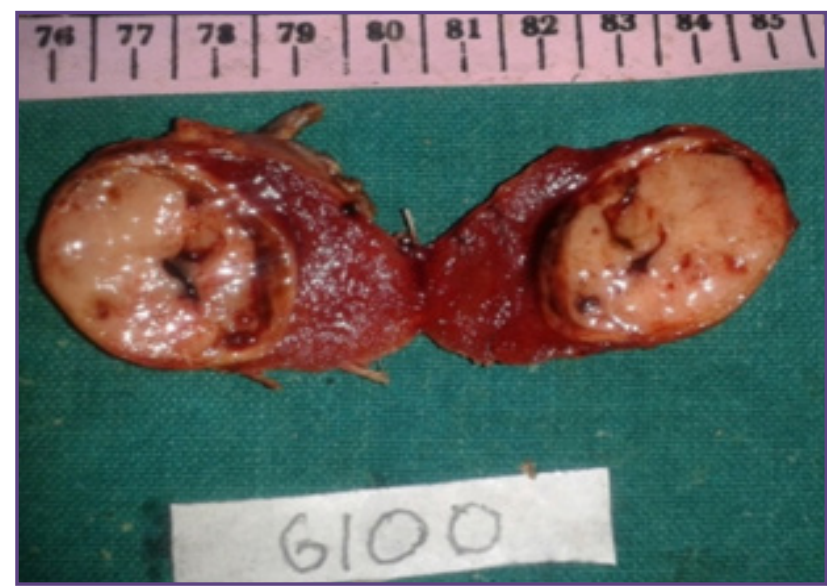

Fig. 1: Gross picture of Follicular neoplasm.

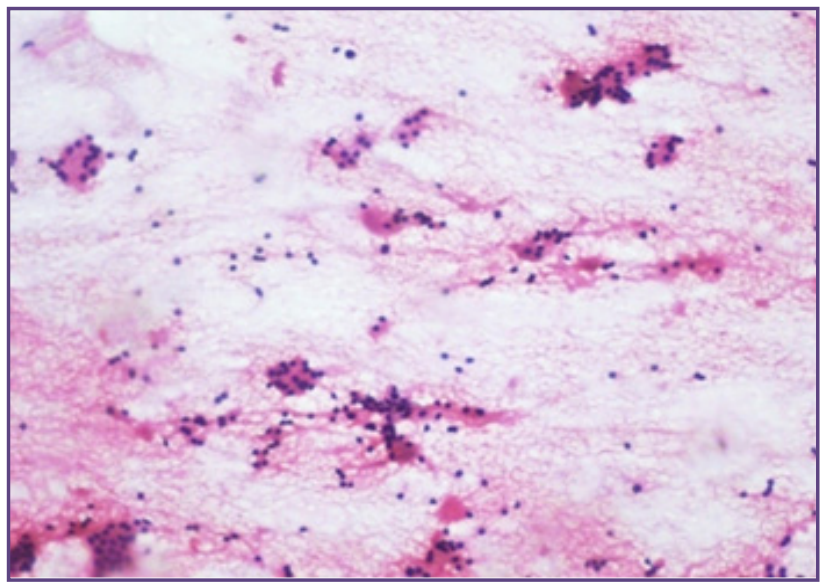

Fig. 2:Follicular neoplasm. Smear shows repeating units of microfollicles[H\&E X100].

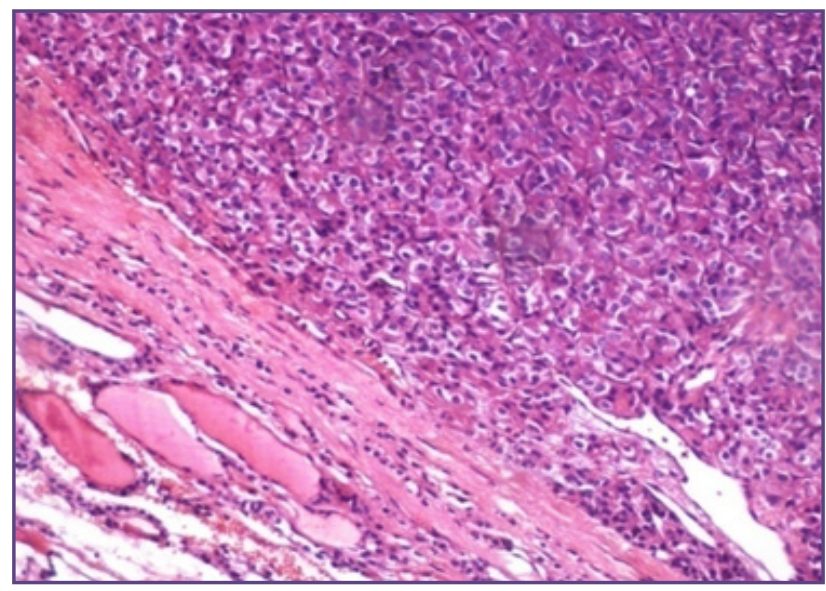

Fig 3:Follicular adenoma. Section shows an encapsulated lesion with compression of surrounding normal thyroid follicles [H\&EX100] 
Table 1:Age and sex wise distribution of the thyroid lesions.

\begin{tabular}{|c|c|c|c|c|}
\hline & Age groups(years) & & \\
\hline Sex & $<20$ & $21-40$ & $41-60$ & $61-80$ \\
\hline Male & 0 & 1 & 1 & 2 \\
\hline Female & 4 & 31 & 18 & 3 \\
\hline
\end{tabular}

Table 2: Analysis of the cyto-histopathological correlation of thyroid lesions.

\begin{tabular}{|c|c|c|c|c|}
\hline \multirow{2}{*}{ Cytological diagnosis } & Histopathological diagnosis & \multirow{2}{*}{ Total } & \multirow{2}{*}{ Comment } \\
\cline { 2 - 5 } & Benign & Malignant & & False negative-2 \\
\hline Benign & 53 & 2 & $\mathbf{5 5}$ & False positive-0 \\
\hline Malignant & 0 & 5 & $\mathbf{6 0}$ & \\
\hline
\end{tabular}

\section{Discussion}

Thyroid FNAC was initially started by Martin and Ellis in 1930. It is usually the first line of investigation and a minimally invasive diagnostic procedure whose essential role is to diagnose and distinguish benign from malignant lesions. A total of 60 cases of thyroid lesions who underwent FNAC with subsequent histopathological examination were included in the study and all the lesions were analysed with their history, FNAC findings and histopathology.

In the present study there was a female predominance with F:M ratio of 14:1.It was similar to the studies done by Ranjan et al. ${ }^{[1]}$ and Tilak et al. ${ }^{[8]}$. Age of the patients ranged from 15-65 years and majority of the patients were in the 21-40 years age group. It is in concordance with the study done by Kumar et al. ${ }^{[9]}$ Out of the 60 cases, Cytologically 55 cases were diagnosed as non-malignant and 5 cases as malignant lesions. Of these 55 cases, 53 cases proved to be non-malignant by histopathology resulting in 2 false negative cases. All 5 cases diagnosed as malignant by cytology were proven to be malignant by histopathology and there was no false positive case in the present study, probably it could be because of small sample size.

When Galen and Gambino method is applied, FNAC of the thyroid lesions had a sensitivity of $96.36 \%$ and specificity of $100 \%$. The overall diagnostic accuracy in detecting malignant tumors was $96.66 \%$. This study is comparable with the other studies as shown in the table 3.
In the present study , 2 cases were erroneous and were false negative cases. One case was diagnosed as Colloid goitre with cystic change by FNAC and it was subsequently diagnosed as cystic papillary thyroid carcinoma by histopathology. The greatest risk of a false negative diagnosis is in relation to cystic neoplasms, mainly cystic papillary carcinoma. Over $40 \%$ of the cystic neoplasms may be missed by FNAC. Amatya et al. ${ }^{[10]}$ and Fernandes $\mathrm{H}$ et al. ${ }^{[11]}$ also found a similar misdiagnosis in their study.

The cause of false negative results were the poorly cellular sample in a cystic papillary carcinoma and the thick fibrous capsule. Gagneten stressed the importance of doing multiple aspirations in a thyroid swelling in order to obtain representative material from different areas since the thyroid can be affected by more than one disease process. ${ }^{[12]}$

The diagnostic error was most commonly due to inadequate specimens and cystic lesions. One must be careful in committing a false negative diagnostic error in cystic lesions that contain macrophages and scanty material, since these features do not exclude malignancy. Repeat FNAC or thyroidectomy is advised for persistent nodules. ${ }^{[13,14]}$ Cystic thyroid lesions pose diagnostic difficulties. Cystic change and/or hemorrhage in neoplasms is seen in upto $25 \%$ of primary papillary carcinomas, In $20 \%$ of follicular neoplasms and in $26 \%$ of follicular carcinomas. ${ }^{[8]}$

Table 3: Comparison of results of present study with previous studies in identifying thyroid malignancies

\begin{tabular}{|l|c|c|c|c|}
\hline Studies & Year & Sensitivity & Specificity & Accuracy \\
\hline Gupta et al. $^{5}$ & 2010 & 80 & 86.6 & 84 \\
\hline Pinky pandey et al. $^{6}$ & 2012 & 57.14 & 90 & 80.28 \\
\hline Parikh et al. $^{7}$ & 2012 & 71.43 & 100 & 90.24 \\
\hline Ranjan et al. $^{1}$ & 2014 & 82.14 & 86.8 & 83.60 \\
\hline Gamit et al. $^{3}$ & 2015 & 92.85 & 98.48 & 97.5 \\
\hline Present study & 2015 & 96.36 & 100 & 96.66 \\
\hline
\end{tabular}


Different studies by Heimann A and Gritsman A suggest that, multiple criteria must be observed before making a confident cytological diagnosis of papillary carcinoma. Logistic regression analysis of various criteria suggested that a combination of a intranuclear cytoplasmic inclusion, papillary structure with or without adherent blood vessels and dense metaplastic cytoplasm were the three most important variables. ${ }^{[15]}$

Commonest lesion encountered in thyroid gland was colloid goitre and most of the cases correlated with the histopathological diagnosis. Of the 45 cases diagnosed as goitre, all were correctly verified except 5 cases which were diagnosed as follicular adenoma. In these cases, the lesions were cystic, colloid material was aspirated, and the smears showed features of nodular goitre. However, on sectioning, there was nodular mass which turned out to be a follicular adenoma. ${ }^{[10]}$ The cytological appearances in colloid goitre form a continuum which merge with those of follicular adenoma, and in this grey area, cytological criteria alone cannot reliably distinguish between the two. $\left.{ }^{[16]}\right]$

2 cases of goitre were misdiagnosed as follicular neoplasm in FNAC, it is a known fact that the cytological criteria cannot reliably differentiate between the two because of the overlap. Smears from micro follicular areas in a nodular goitre may show a repetitive pattern of micro follicles or rosettes similar to the follicular neoplasm, and if only such a focus is aspirated and sampled, the differentiation from follicular neoplasm is impossible. ${ }^{[15]}$ In the present study similar type of microfollicular areas were sampled and lead to the erroneous diagnosis of follicular neoplasm. Another false negative case was a follicular carcinoma which was misdiagnosed as colloid goiter by FNAC. The differentiation of thyroid adenoma from follicular carcinoma based on cytologic criteria done is difficult and challenging as the cytological appearences of both are very similar. Lowhagen advocated that a cytologic report should only state that a follicular neoplasm is present with no implications of its benign or malignant nature. Friedman advised histologic examination in such cases for final diagnosis. ${ }^{[17]}$

Thus FNAC is an efficient diagnostic procedure for assessing the thyroid lesions. The method is simple, safe and economical. Another advantage is that it is a time saving procedure which can be reported within minutes and hours instead of days needed for histopathology studies. Nevertheless, the specificity of $100 \%$, sensitivity of $96.36 \%$, and an overall diagnostic accuracy of $96.66 \%$ are very encouraging. These results show a strong correlation of FNAC with the histopathology of the thyroid lesions.
FNAC should be regarded as an extremely valuable complement to the conventional surgical histopathology and is just indispensible.

\section{Conclusion}

FNAC serves as a rapid, convenient and an accurate outpatient method for diagnosis of palpable thyroid lesions than any other combination of clinical laboratory tests. There is almost perfect cytohistopathological concordance with a sensitivity of $96.36 \%$ and specificity of $100 \%$ as evaluated in this study. These results are consistent with those available in the literature. It also helps as a guide for appropriate therapeutic management to either locally excise a benign tumor or plan for a radical surgery in case of malignancies. Hence, we conclude that FNAC serves as a first line investigation for palpable thyroid lesions and acts as a useful screening test to triage thyroid lesions before surgery.

\section{References}

1. Agrawal R, Saxena M, Kumar P. A Study of Fine Needle Aspiration Cytology of Thyroid Lesions with Histopathological Correlation. Indian Journal of Pathology and Oncology.2015;2(4):277-283

2. Bakhos R, Selvaggi SM, DeJong S, Gordon DL, Pitale SU, Herrmann M and Wojcik EM. Fine-needle aspiration of the thyroid: Rate and causes of cytohistopathologic discordance. Diagn. Cytopathol.2000;23(4): 233-237

3. Gamit MJ, Talwelkar SR, Dhruva GA. Histocytological Correlation Study of Thyroid Gland Lesions.International Journal of Science and Research. Nov 2015;4(11):777-780.

4. Yassa L, Cibas ES, Benson CB, Frates MC, Doubilet PM, Gawande AA et al. Long-term assessment of a multidisciplinary approach to thyroid nodule diagnostic evaluation. Cancer Cytopathology. 2007 Dec 25;111(6):508-16.

5. Gupta M, Gupta S, Gupta VB. Correlation of fine needle aspiration cytology with histopathology in the diagnosis of solitary thyroid nodule. Journal of thyroid research. 2010 Apr 18;2010.

6. Pandey P, Dixit A, Mahajan NC. Fine-needle aspiration of the thyroid: A cytohistologic correlation with critical evaluation of discordant cases. Thyroid Research and practice. 2012 May ;9(2):32-39

7. Parikh UR, Goswami HM, Shah AM, Mehta NP, Gonsai RN. Fine needle aspiration cytology (FNAC) study of thyroid lesions (study of 240 cases). GMJ. 2012;67(2):25-8.

8. Tilak V. Dhaded AV, Jam R. Fine needle aspiration cytology of head and neck masses. Indian J Pathol Microbiol. 2002 Jan; 45(1):23-9.

9. Kumar SK, Seetharamaiah T, Rampure D, Ramakrishna C, Devi RY. Thyroid nodule: Cytohistological correlation. Scholar J Appl Med Sci.2013;1(6):745-747 
10. Amatya BB, Joshi AR, Singh SK, Panth R, Basnet RB. A study of fine needle aspiration cytology of head and neck masses and their corroboration by histopathology. Post graduate medical journal of national academy of medical sciences. 2009;6(2).

11. Fernandes H, D'Souza CRS, Thejaswini BN. The role of fine needle aspiration cytology in palpable head and neck masses. Journal of clinical and diagnostic research. 2009 Oct; (3):1719-1725.

12. Gagneten CB, Roccataglinta G, Lowenstein A et al. The role of fine needle aspiration biopsy cytology in the evaluation of the clinically solitary thyroid nodule. Acta Cytologica. 1987;31:595-598

13. Hsu CH, Boey J, Diagnostic pitfalls in the fine needle aspiration of thyroid nodules. A study of 555 cases in Chinese patients. Acta Cytologica.1986Dec;31(6): 699-704.

14. Goellner JR, Gharib H, Grant CS, Johnson DA. Fine needle aspiration cytology of the thyroid,1980 to 1986.Acta Cytologica.1986Dec;31(5): 587-90

15. OreIl SR, Sterrett GF, Walters MN-I, Whitaker D. Manual and atlas of fine-needle aspiration cytology, The thyroid gland. Third edition : Churchill Livingstone, 2003: 110 -140.

16. Orell SR, Sterrett GF, Whitaker D et al: The thyroid gland. In Manual and atlas of fine- needle aspiration cytology, 2nd Edn: Churchill Livingstone, 1992:102-104

17. Lowhagen T, Sprenger E. Cytologic presentation of thyroid tumors in aspiration biopsy smear. A review of 60 cases. Acta Cytologica.1974;18(3):192-197.

*Corresponding author:

Dr Shakuntala S Aramani, Assistant Professor, Department of Pathology, Gadag Institute of Medical Sciences, Mallasamudra, Gadag-582103.Karnataka, India. Phone: +91 9663935998

Email: shakkuaramani@gmail.com

Date of Submission : 11.12.2016

Date of Acceptance : 03.03.2017

Financial or other Competing Interests: None.

Date of Publication : 04.06.2017 